Ministerstwo Nauki

i Szkolnictwa Wyższego

Digitalizacja archiwalnych numerów czasopisma naukowego Analecta Cracoviensia 1-24 (1969-1992) i ich publikacja w otwartym dostępie - zadanie finansowane w ramach umowy 672/P-DUN/2017 ze środków Ministra Nauki i Szkolnictwa Wyższego przeznaczonych na działalność upowszechniającą naukę

ANDRE GOURON

Université de Montpellier I

Faculté de Droit et des Sciences Economiques

\title{
DOCTRINE MEDIEVALE ET JUSTICE FISCALE: PIERRE ANTIBOUL ET SON TRACTATUS DE MUNERIBUS
}

L'attention a été récemment attirée ${ }^{1}$ sur la figure et l'oeuvre d'un juriste jusque là bien oublié, Pierre Antiboul. Celui-ci est pourtant l'auteur d'un Diversorium ou Tractatus de muneribus dont on connaît au moins deux éditions incunables ${ }^{2}$ et qui a été inséré au Tractatus universi juris ${ }^{3}$. Les lignes qui suivent, consacrées à ce Provençal formé au studium montpelliérain et dont l'oeuvre, nourrie de droit savant, n'en manifeste pas moins une vive originalité, ne sauraient être mieux dédiées qu'à notre éminent collègue Adam Vetulani dont les si précieuses rechercres font de lui l'un des représentants les plus marquants de cette école historique polonaise de laquelle sont issus tant d'études médiévales de grande qualité.

Si la date de naissance d'Antiboul est inconnue, ses origines sont assurées: il indique que son père était natif d'Hyères, et que lui-même a vu le jour au Cannet, près de Draguignan, ville dont il se considère comme incola lorsqu'il rédige son traité, ce qui suppose, d'après la définition qu'il donne de ce terme, qu'il y réside alors depuis plus de dix ans ${ }^{4}$. Pourtant sa vie ne s'est pas écoulée sans qu'il quitte la Provence. Il a étudié le droit à Montpellier, d'où il tire le seul mâtre qu'il se reconnaisse, Brémond de Montferrier ${ }^{5}$. La référence est malheureuse-

1 D. Maffei, Qualche postilla alle ricerche di E. M. Meijers sulle scuole di Orléans, Tolosa e Montpellier, "Tijdschrift voor Rechtsgeschiedenis”, t. XXXVI (1968) p. 389 et n. 4 .

2 Lyon, 1493 (Hain Copinger, 1146) et 1498 (Pellechet, 803).

3 Ed. Venise, 1584 , t. XII, fos $19-51$ vo. Les références qui suivent se rapportent à cette édition.

${ }_{4}$ Tract. IV. 4 , § 91 , fo 45 , c. 1 .

5 Tract. IV. 4 , § 26 , fo 40 vo, c. 2. 
ment de chronologie un peu imprécise, car Brémond a professé à Montpellier depuis 1279 et peut-être jusqu'à sa mort en 1307, sauf des interruptions sans doute assez brèves ${ }^{6}$. Quant aux doctores Bononienses, il s'y réfère, selon la formule classique, ut audivi ${ }^{7}$.

Une chronologie un peu plus serrée peut être tirée de la comparaison entre les auteurs cités, d'une part dans le Tractatus d'Antiboul, d'autre part dans l'oeuvre beaucoup plus connue d'un autre élève de Brémond, l'Aurea practica libellorum, de Pierre Jame (Petrus Jacobi). Ce dernier est vraisemblablement arrivé à Montpellier en 1290, où il a été étudiant et presque sûrement professeur; sa Practica a été écrite en plusieurs étapes de 1311 à 1329 , sans compter quelques additions postérieures ${ }^{8}$. Il vivait encore en 1351, mais un document de 1367 le donne pour décédé ${ }^{9}$, et sa mort, à notre avis, n'est guère postérieure à la première de ces deux années.

La formation de Pierre Jame et celle de Pierre Antiboul présentent certes des points communs: outre Brémond, ils ont tous deux suivi l'enseignement donné par Hugues Roger ${ }^{10}$, un autre Montpelliérain dont la carrière est fort mal connue. Et l'influence des doctores tholosani se traduit chez l'un comme chez l'autre par des renvois à Bertrand de Scandalis et à Guillaume de Ferrières. Mais nous serions enclin à considérer Antiboul comme le cadet de Jame, qui a eu aussi pour maîtres Guillaume Accurse, trés probablement professeur à Montpellier vers $1290^{11}$, et Guillaume de Plaisians, dont la carrière enseignante a cessé au plus tard en 1301, lors de sa nomination en qualité de juge mage de la sénéchaussée de Beaucaire-Nîmes ${ }^{12}$. Non seulement Antiboul ne les cite pas, mais encore ses références concernent des professeurs montpelliérains un peu plus jeunes: Oldrade, qui, après avoir enseigné à $\mathrm{Pa}-$ doue de 1307 à 1310 , est vraisemblablement venu à Montpellier dont il cite la coutume ${ }^{13}$, Bertrand de Deaux, dont les opinions sont presque toujours associées à celles d'Hugues Roger, et qui a sûrement professé

6 Pour la vie de Brémond, nous nous permettons de renvoyer aux pages que nous avons consacrées à ce juriste dans les Etudes [...] Edouard Baratier, Marseille 1974

7 Tract. IV. 4 , § 30 , fo 41 , c. 2.

8 Cf. Paul Fournier, Pierre Jame (Petrus Jacobi) d'Aurillac, jurisconsulte. Histoire Littéraire de la France, t. XXXVI (1927) p. 481-521.

9 P.-F. Fournier, Nouveaux documents sur le jurisconsulte Pierre Jacobi et sa famille, „Bibl. Ecole des Chartes”, t. XCVIII (1937) p. 223.

10 Le fait avait été relevé, à propos d'Antiboul, par R. Caillemer, Lo Codi et le droit provençal au XIIe siècle, "Annales du Midi”, t. XVIII (1906) p. 499.

$11 \mathrm{H}$. Gilles, Accurse et les Universités du Midi de la France. Atti del convegno internazionale di studi accursiani, t. III, Milan 1968, p. 1039-1040.

12 A. Henry, Guillaume de Plaisians, ministre de Philippe le Bel, „Le Moyen-Age", février 1892, p. 34.

13 Ceci ressort notamment du cons. 192; cf. pourtant les hésitations manifestées par E. Will, Die Gutachten des Oldradus de Ponte zum Prozess Heinrichs VII. gegen Robert von Neapel, Berlin-Leipzig 1917, p. 7. 
au même studium, mais sans doute postérieurement à $1305^{14}$, et enfin Bernard Sabors, encore simple jurispérit en $1313^{15}$. Nous verrions ainsi volontiers les années d'études d'Antiboul s'inscrire dans la première décennie du quatorzième siècle. Les plus récents des auteurs qu'il cite ont peut-être été ses collègues: en effet, si aucun document, à notre connaissance, ne témoigne directement de son activité professorale, le Tractatus consacre de longs développements aux privilèges fiscaux des professeurs - en même temps du reste que des médecins, selon une tradition bien montpelliéraine - et à une comparaison entre licence et doctorat; il n'y manque pas le rappel classique des textes conférant le titre de comes à celui qui a enseigné pendant vingt ans ${ }^{16}$. Et que son auteur porte le plus souvent le simple titre de jurispérit n'exclut nullement quelques cours aux heures extraordinaires.

Ce que nous savons de sa vie ne peut être tiré, indépendamment de son oeuvre, que de quelques documents exclusivement provençaux, d'où l'on n'obtient rien d'assuré sur la période de sa formation juridique. Ainsi Antiboul figure en qualité de témoin dans un compromis passé le 11 juillet 1303 entre les habitants du Cannet et les deux seigneurs du lieu ${ }^{17}$, ainsi qu'au titre de délégué du juge ordinaire de Draguignan, la même année, lorsque les représentants de la communauté de Fayence en appellent de la contribution aux fouages qui leur est imposée ${ }^{18}$.

Gagna-t-il Montpellier avant ou après 1303? Il n'est guère possible de se montrer affirmatif à travers les éléments bibliographiques rassemblés par l'érudit dracénois Fréd. Mireur ${ }^{19}$. Notre juriste n'y apparaît qu'une fois pendant le quart de siècle qui suit: il rend le $1^{\mathrm{er}}$ novembre 1308 un arbitrage entre le prieur de Palayson, Adhémar de Grignan, et les syndics de Roquebrune, en compagnie de l'illustre Jean Caba-

14 E. M. Meijers, Responsa doctorum tholosanorum, Haarlem 1938, p. IV et XXVII-XXVIII. L'année 1305, au cours de laquelle il obtient l'église de Saint Niarcel de Carreiret, près d'Uzès, fournit sans doute le point de départ de son séjour montpelliérain, attesté d'autre part à travers la connaissance de la ville qu'il manifeste dans ses gloses.

15 La carrière de ce professeur a été résumée par Meijers, op. cit., p. IV, n. 1, non sans une légère inexactitude: il ressort des listes d'officiers consulaires publiées par Al. Germain, Histoire de la commune de Montpellier, t. I, Montpellier 1851 , p. 390 et s., que Bernard est devenu docteur entre 1313 et 1321.

16 Tract. III, § 51 et s., fo 25 , c. 2 ; $\$ 56$ et s., fo 25 vo, c. 1.

17 Arch. Mun. Le Cannet, AA 4.

18 Arch. Mun. Fayence, CC 22.

19 Les rues de Draguignan et leurs maisons historiques, t. VI, Draguignan 1924 , p. $11-15$. 
ssole ${ }^{20}$, qu'il déclare du reste dans son traité avoir vu en sa présence rendre un consilium à Aix à propos de fouages ${ }^{21}$.

Très vraisemblablement, Pierre Antiboul a consacré l'essentiel de sa carrière à exercer comme avocat à Draguignan. Son traité est truffé d'allusions ou de références aux consultations qu'il a données aux habitants de la cité et des localités voisines. Il est d'autre part qualifié de jurispérit de cette ville lorsque le sénéchal de Provence le désigne, en mai 1329 , pour enquêter sur les agissements des officiers royaux dans un certain nombre de vigueries et de bailliages ${ }^{22}$. On le retrouve encore à Draguignan en 1333, lors de la venue de l'archiprêtre de Bénévent, Léopard de Fulginet, chargé d'une nouvelle enquête ${ }^{23}$.

D’après Mireur, Antiboul aurait résidé à Marseille de 1336 à 1340 , ce qui s'accorderait à l'opinion de Ruffi, qui le donne pour juge du palais dans cette ville. En tout cas, sa trace semble perdue par la suite. Assurément, la tradition qui le fait mourir en 1389, telle que nous la transmet Papon ${ }^{24}$ et que la reprennent des auteurs modernes ${ }^{25}$, malgré des réserves dès longtemps exprimées ${ }^{26}$, repose sur une erreur: Antiboul était mort avant le 5 juin 1357, date à laquelle ses héritiers interviennent dans un acte notarié; sa veuve lui survivait en 1376 encore, alors que ses deux fils, dont le deuxième lui avait succédé comme avocat avant de devenir juge de Hyères, étaient disparus depuis cinq ans au moins ${ }^{27}$.

A peu de choses près ${ }^{28}$, il ne nous est parvenu, de l'oeuvre d'Antiboul, que la version imprimée de son traité de muneribus; l'auteur indique

20 Sur ce célèbre professeur à Avignon et à Naples, et sur les nombreuses missions administratives et diplomatiques qui lui furent confiées, cf. en dernier lieu G. Giordanengo, Consultations juridiques de la région dauphinoise (XIIIe-XIVe siècles), „Bibl. de l'Ecole des Chartes”, t. CXXIX (1971) p. 56-57 et M. Hayez, Dizionario biografico degli Italiani, t. XV (1972) p. 676-678. On a gardé de lui un certain nombre de sentences arbitrales: cf. Arch. Dép. Vaucluse, G. 5, fo 20 et Arch. Mun. Cavaillon, DD 2 et 3, etc. Sur son activité comme maître rational de Provence, cf. Encyclopédie des Bouches-du-Rhône, t. II, Paris - Marseille 1924, p. 618.

${ }_{21}$ Tract. IV. 2 , § 49 , fo 35 , c. 2 .

22 Arch. Dép. Bouches-du-Rhône, B 2047, fos 42 et s.

23 Ibid., B 1065. Sur ce document, cf. Ed. Baratier, Enquêtes sur les droits et revenus de Charles Ier d'Anjou en Provence (1252 et 1278), Paris 1969, p. 20 et p. 25.

24 Histoire générale de Provence, t. III, Paris 1784, p. 478.

25 Aug. Brun, La littérature en langue latine, dans Encyclopédie des Bouches-du-Rhône, t. II, p. 796; P. Masson, Dictionnaire biographique des origines à 1800 (ibid., t. IV, vol. II), Marseille 1931, p. 27.

${ }_{26}$ Achard, Dictionnaire de la Provence et du Comté-Venaissin, t. I, Marseille 1786, p. 28.

${ }_{27}$ Sur tous ces points, cf. Fréd. Mireur, loc. cit.

28 Ainsi le ms. Vat. lat. 5773, fos 51 et s.: cf. M. Bevilacqua, Una „quaestio" di Niccoló Matarelli (Vat. lat. 10726). Collectanea... Anselmi M. Card. Albareda..., t. I, Cité du Vatican 1962 (Studi e testi, 219), p. 145. 
lui-même avoir pourtant rédigé deux autres traités, de moneta et de consuetudine ${ }^{29}$, qui semblent avoir disparu. Le de muneribus qu'aucun indice direct ne permet de dater ${ }^{30}$ est à n'en pas douter une oeuvre de maturité, sinon de vieillesse: l'abondance des exemples tirés de consultations antérieures suppose une expérience professionnelle approfondie, tandis que, comme on l'a vu, la qualité d'incola suppose une installation déjà ancienne à Draguignan. Peut-on être plus précis en utilisant les circonstances d'affaires parfois décrites? S'il y a peu à tirer, malgré les apparences, de l'expression tempore meo à propos d'une exemption fiscale accordée par Raimbaud de Simiane à des hommes vivant sur des terres constituées en dot par sa femme, Marcelle de Pontevès ${ }^{31}$, on peut espérer plus avec le récit d'un conflit opposant les habitants du Cannet aux héritiers de Guillaume Puget, anobli par Arnaud de Villeneuve sur concession du comte de Provence ${ }^{32}$. Malheureusement l'anoblissement remonte à 1293 , et on ignore la date du décès du bénéficiaire ${ }^{33}$.

Les auteurs qui ont la prédilection d'Antiboul donnent une idée un peu plus juste: les plus récents sont ceux d'un écrivain vivant à la fin $\mathrm{du}$ premier tiers du quatorzième siècle ou peu après. Outre Revigny et Belleperche, on y trouve, à côté de Jean d'André et d'Oldrade, les.Français Guillaume de Cunh et Bertrand de Deaux; surtout, parmi les références les plus récentes, reviennent fréquemment les noms de Bernard Sabors, dont les gloses n'ont guère pu circuler avant 1325, de Raymond Faure, professeur toulousain autour de 1328, de Bertrand de Scandalis qui, professeur in utroque, est donné pour juge de la Cour commune de Rodez en 1333, et d'Arnaud Escarbot, qui vivait encore en $1335^{34}$. D'un autre côté, la rédaction du Tractatus a eu lieu à une époque où le comté de Provence est, ou était encore récemment, aux mains d'un homme, mais où se pose la question de savoir si le mariage d'une petite-fille de la famille comtale entraîne le paiement d'un fouage ${ }^{35}$. Il s'agit donc,

29 Tract. IV. 2 , $\S 78$, fo 37 , c. 1 ; IV. 4 , $\S 10$, fo 40 , c. 1 .

30 César de Nostredame, qui fait un long et vibrant éloge du traité d'Antiboul dans sa Chronique de l'histoire de Provence (éd. Lyon 1614, p. 336-337), le place à hauteur des années 1316-1318, mais n'en donne aucune justification.

31 Tract., IV. 4, $\S 153$, fo 48 , c. 2; nous remercions M. Fr.-P. Blanc, maître-assistant à la Faculté de Droit et des Sciences Politiques d'Aix, qui a bien voulu nous signaler que Raimbaud s'était marié après 1242 et testa en 1270 .

32 Tract., IV. $4, \S 135$, fo 47 , c. 1.

33 Qu'Arnaud soit donné pour déjà mort au temps de la rédaction du Tractatus porte toutefois cette dernière au-delà de 1303; mais il n'est pas possible de le confondre avec un autre Arnaud, l'illustre général de la reine Jeanne, qui vivait encore en 1368: cf. sur ce dernier Fr.-P. Blanc, Origine des familles provençales maintenues dans le second ordre sous le règne de Louis XIV. Dictionnaire généalogique, th. Droit, Aix 1971, p. 582-583. - Parmi les hértiers mentionnés au texte, figurait sans doute ce Guillaume Puget à qui Louis de Tarente inféode le château de Figanières en 1349: Arch. Dép. Bouches-du-Rhône, B 6, fo 134 .

${ }_{34}$ Cf. E. M. Meijers, Responsa.., p. XLII et s.

35 Cf. Tract. IV. $2, \S 76$, fo 37 , c. 1 . 
soit des années comprises entre 1328 et 1343, sous le règne de Robert, mais après la mort de son fils Charles de Calabre, la succession ne pouvant échapper à l'une des petites-filles de Robert, soit des tout premiers temps du règne de Jeanne. Ainsi Antiboul a dû rédiger l'essentiel de son oeuvre autour de 1340 , peut-être même en 1344, lorsque la perception d'un fouage à l'occasion du mariage de Marie, soeur de la reine Jeanne, avec Charles de Duras provoqua une grande agitation dans la province ${ }^{36}$; quelques adjonctions postérieures, du reste, ne sont pas à exclure.

Imprégné, comme on le voit, de la science des docteurs méridionaux, parmi lesquels il privilégie Montferrier, Hugues Roger et Bertrand de Deaux, souvent associés, pour les Montpelliérains, Guillaume de Ferrières et Escarbot pour les tholosani, qu'il vise parfois sous cette dernière forme, Antiboul fait preuve d'autre part d'une culture juridique étendue: innombrables sont ses renvois à Cino de Pistoie, à Dino de Mugello, à Jacobus de Arena, à Albertus Gandinus, sans parler de l'utilisation qu'il fait de Martinus de Fano, de Guido de Suzaria, voire d'Hubertus de Bobbio. De tous les canonistes auxquels sont faits de larges emprunts, ressortent surtout Hostiensis, le Spéculateur et Jean d'André. L'opinion de notre Provençal est volontiers personnelle: s'il préfère souvent les arguments de Bertrand de Deaux à ceux d'Hugues Roger, il n'hésite pas à s'écarter du premier, par exemple, pour rallier Raymond Faure ${ }^{37}$. D'une manière plus générale, Pierre Antiboul fait preuve d'une raisonnable indépendance. Dès sa préface, il critique de manière à peine voilée la tendance de ses contemporains à préférer les discussions théoriques aux questions pratiques ${ }^{38}$ et son ironie s'exerce volontiers sur les querelles de doctrine trop poussées, par exemple à propos du brocard qui tacet ${ }^{39}$; et si la glose est massivement utilisée, il l'écarte parfois ${ }^{40}$, mais tente le plus souvent de la justifier, par une attitude bien montpelliéraine, ou du moins commune aux élèves de Brémond de Montferrier ${ }^{41}$. De même, le Speculum est fréquemment allégué, mais ce n'est pas toujours avec beaucoup de respect: son auteur est accusé de trancher transiens sicco pede, voire pis encore ${ }^{42}$.

Le plan de l'oeuvre n'est pas sans défauts; il est vrai que, comme

36 Cf. E.-G. Léonard, La jeunesse de Jeanne Ière, reine de Naples, comtesse de Provence, Th. Lettres, Paris. Monaco-Paris 1932, t. II, p. 59.

37 Tract. II, § 9 , fo 22 , c. 2 .

38 Tract., $\mathrm{f}^{\circ}$ 19, c. 2 : ,[...] legibus certis et theoricae juris potius operam dantes quam circa facta incerta jurisque practicam intendentes [...]".

${ }_{39}$ Tract. IV. 4, $\S 171$, fo 49 , c. 2 : ,,[...] invenies in ista materia pluviam abundantem, et de mari et vento copiam [...]".

40 Cf. par exemple Tract. III, § $90, f^{\circ} 27 \mathrm{v}^{\circ}$, c. 2.

41 Voir sur ce point H. Gilles, op. cit.

42 Tract. IV. 4 , $\S 207$, fo 51 vo, c. 1 ; cf. aussi ibid., $\S 90$, fo 45 , c. 1 : „,...] ego credo quod Spe. ibi somniavit, aut dormitabat, aut biberat, aut ego ipsum non intelligo [...]". 
Antiboul le remarque dans sa préface, la matière était dispersée au point de justifier le titre de Diversorium. Une répartition en quatre parties entraîne toutefois un bien inégal découpage, puisque les deux premières intitulées de personis munera subeuntibus et de modo munerum, représentent au total moins de la moitié de l'étendue propre à la troisième, de excusatione munerum; mais que dire surtout de la quatrième, annoncée comme un simple supplément groupant diversae quaestiones super muneribus, et qui forme finalement à elle seule les deux tiers de l'ouvrage? Nous croirions volontiers que ces quaestiones, aux solutions traditionnellement empreintes de doute selon l'auteur, ont été rédigées à un autre moment que les développements qui les précèdent, ou au moins que les deux premières parties; ainsi s'expliqueraient non seulement l'anomalie de leurs dimensions respectives, mais aussi les différences de style: les allégations d'auteurs, si nombreuses dans les trois premières parties, se font ensuite beaucoup plus rares, tandis que les observations personnelles sur la pratique fiscale et même sur le tissu social, nourries d'une foule d'exemples, prennent une ampleur nouvelle, témoignant d'une âpreté accrue dans la critique, notamment à l'égard de la seigneurie provençale contemporaine. Au reste le plan de cette quatrième partie n'est guère rationnel, car il comporte quatre chapitres dont le deuxième et le troisième portent sur les classifications des impôts, tandis que le quatrième, beaucoup plus long - il couvre plus du tiers de l'ouvrage complet - traite pêle-mêle des exemptions et immunités fiscales, des privilèges nobiliaires, des actions, des droits du vassal sur son fief, des officiers seigneuriaux, etc.

Oeuvre d'un praticien, le Tractatus s'adresse à des praticiens: Antiboul estime devoir porter conseil, non seulement à ses lecteurs, mais aussi aux clients de ces derniers ${ }^{43}$. Toutefois, une culture juridique très supérieure à la moyenne l'amène à adopter des positions de principe volontiers nuancées; si par exemple le recours à la coutume est largement admis, comme il se doit en la matière ${ }^{44}$, la consuetudo irrationabilis est écartée à plusieurs reprises, et tout particulièrement lorsqu'elle heurte le droit canonique ${ }^{45}$. Malheureusement, l'auteur renvoie fréquemment au traité perdu de consuetudine, et il se contente souvent de quelques lignes critiques visant des règles qui lui paraissent contestables, comme les privilèges fiscaux des milites ${ }^{46}$. Dans l'ensemble, et sans

\footnotetext{
${ }_{43}$ Tract. IV. 4 , § 71, fo 44, c. 1: ,[...] scias te defendere et clientulos tuos [...]".

44 Tract. IV. 2, § 10 , fo 33 , c. 2 : ,[...] quae consuetudines in hac materia munerum considerandae sunt et servandae [...]".

${ }_{45}$ Tract. III, § 117 , fo $29 \mathrm{vo}$, c. 2.

46 Tract. IV. 4, § 10 , fo 40 , c. 1 , ,[...] quia consuetudo hujusmodi regionis tenet milites nostri temporis francos, sed tamen in ista consuetudine inducenda et probanda pendent leges et prophetae, et hic labor, hoc opus est, ut plene dixi in tractatu meo [...]".
} 
doute sous l'influence de Brémond de Montferrier, notre Provençal manifeste une révérence marquée vis-à-vis des sources canoniques, et son attitude à l'égard du clergé local, auquel il épargne ses flèches, reste volontiers favorable. Tout au plus professe-t-il une vive hostilité à l'encontre de l'immunité fiscale per omnia dont jouissent alors les clercs mariés ${ }^{47}$.

L'intérêt essentiel du Tractatus tient à la méthode utilisée pour décrire la matière fiscale: au long de développements purement techniques, s'insèrent des notations de caractère social qui donnent à l'ouvrage une coloration personnelle très forte. Les premiers, qui sont justifiés par la fiscalité des angevins, ,pointilleuse et tracassière, lourde et envahissante" ${ }^{48}$, résument une théorie traditionnelle et menacée, où le feu reste réel et ne constitue pas encore cette unité théorique de répartition qu'il deviendra rapidement dans le royaume de France ${ }^{49}$. La summa divisio entre impôts personnels, mixtes et patrimoniaux - ces derniers se répartissant entre ceux qui restent purement patrimoniaux et ceux qui sont établis personis pro rebus - est classique ${ }^{50}$, mais donne l'occasion de fournir un certain nombre d'exemples. Ainsi de la ville de Montpellier, où l'on impose contre leur gré les détenteurs de biens fonciers, même s'ils habitent un village des environs comme celui de Lattes: le munus étant patrimoniale, une telle solution serait justifiée chaque fois qu'il couvrirait une dépense d'utilité publique, comme l'entretien des voies publiques. Mais il y faudrait l'intervention de la loi ou du prince: faute d'un privilège accordé en la matière à la ville, l'imposition manque de fondement juridique ${ }^{51}$. Autre exemple: toujours à Montpellier, la ville lève une taille pour aménager des sources et $\mathrm{y}$ astreint des nobiles et populares de Lunel qui y disposent de possessions. Là encore, bien que les nobiles ne puissent arguer d'aucun privilège et que l'imposition ait un caractère de nécessité publique, la levée paraît irrégulière à Antiboul, en raison à la fois du caractère extraordinaire de ce prélèvement personis pro rebus dont les étrangers sont donc exempts, et de l'éloignement des fonds des Lunellois par rapport à ces sources: contribuere debent in eis tantum qui inde sentiunt utilitates 52 .

Les droits levés à la circulation ne sont pas épargnés par notre juriste ${ }^{53}$ : illicites dans leur principe, ils ne sauraient être fondés que sur

47 Tract. IV. 4 , § 37 , fo 41 vo, c. 1 .

48 V. L. Bourrilly et R. Busquet, La Provence au Moyen-âge, Paris-Marseille 1924 (Encycl. des Bouches-du-Rhône, t. II), p. 82.

49 Tract. IV. 2 , § $55-56$, fo $35 \mathrm{v}^{\circ}$, c. 1 .

50 On la trouve également chez Pierre Jame, Aurea Practica... éd. Lugd., 1511, fos $85 v^{\circ}-87$.

51 Tract. IV. 1 , § 1 et s., fos 30 , c. $2-30$ vo, c. 2 .

52 Tract. IV. $1, \S 21$, fo 31 , c. 1 .

53 Les usurpations en matière de péages étaient du reste fréquentes en Provence à la fin du XIII siècle: cf. Th. Sclafert, Cultures en Haute-Provence. Déboisement et pâturages au Moyen-Age, Paris 1959, p. 70, n. 3. 
une concession du roi ou du prince ou sur une coutume invétérée: ce n'est le cas, ni de la barre levée à Montpellier sur les entrées et sur le trafic de transit ${ }^{54}$, ni de la taxe à laquelle sont soumis à Draguignan les étrangers à la ville pour le blé apporté aux fours et aux moulins, ni des leudes ou des impôts sur le sel que tenteraient d'exiger le comte de Provence ou le Dauphin, ni plus généralement d'un vectigal créé en dehors des concessions précitées ${ }^{55}$.

Bien souvent, Antiboul nous renseigne au passage sur les fraudes fiscales les plus fréquentes: par exemple celle que commettent les frères ou les neveux d'un clerc disposant de biens en commun avec ce dernier, et qui lui cèdent fictivement leur part pour bénéficier de l'immunité, ou encore si le donateur laïc reste en possession des biens donnés ou les reprend du donataire en location ${ }^{56}$. La malitia est dans ce domaine inépuisable, témoin la pratique par laquelle le père d'un clerc, en l'émancipant, lui fait don de tous ses biens: le juriste, qui l'a rencontrée frequenter de facto, l'a déconseillée à ses clients et propose à ses lecteurs d'agir de même ${ }^{57}$.

De beaucoup, les passages les plus intéressants du Tractatus sont constitués par les observations de caractère social. Au premier rang de celles-ci, tant par leur abondance que par leur sévérité, il faut placer les critiques formulées à l'encontre des milites provençaux. Certes la ,,petite noblesse rurale de la province apparaît nombreuse et peu fortunée" 58; mais rarement aura-t-on vu juriste exprimer autant de doléances à l'égard de la classe nobiliaire. D'abord celle-ci n'a plus aucune justification à fournir à l'appui de ses privilèges fiscaux: les leges ne les accordent qu'à ceux qui combattent, et non aux milites nostri temporis qui ne quittent pas leur domicile ${ }^{\mathbf{5 9}}$. D'autre part les concessions de noblesse sont recherchées à des fins purement fiscales, et l'on sent à ce sujet qu'Antiboul les juge abusivement nombreuses, sans aller jusqu'à formuler une critique précise à l'encontre de la politique comtale; du moins tient-il pour sans effet sur la matière l'astucia de l'anoblissement à l'article de la mort afin d'en procurer le profit aux héritiers. Un Dracénois ne pouvait d'ailleurs qu'être sensibilisé à ce pro-

54 Dénoncée aussi par Jame, mais de manière impersonnelle (éd. cit., fo 86 vo).

55 Tract. IV. 4 , § 42-45, fo 42 , c. 1 . - Au lien établi entre souveraineté et imposition indirecte, les années languedociennes d'Antiboul ne sont peut-être pas étrangères: sur les principes alors couramment admis par l'administration royale, cf. J. R. Strayer, Notes on the origin of english and french export taxes, ,Studia Gratiana", t. XV (Post Scripta) 1972, p. 417 et s.

56 Tract. IV. 4 , § $68-70$, fo 43 , c. 2 .

57 Tract. IV. 4 , § 72 , fo 44 , c. 1 .

58 Ed. Baratier, Enquêtes..., p. 69.

59 Tract. IV. 4, § 7, fo 39 vo, c. 2: „[...] Immunitas [...] denegari debet istis militibus non veris sed vanis, quorum militiam non video alicujus utilitatis nec fructus, sed dumtaxat vanitatis et superbiae [...]". 
blème, car un certain nombre de tentatives d'usurpation s'étaient manifestées dans la région ${ }^{60}$.

$\mathrm{Au}$ niveau des principes, l'ouvrage ne remet certes pas en cause le droit féodal, pour le dominus depauperatus, d'obtenir l'aide: les expensae intolerabiles et graves que peut entraîner la chevalerie, la rançon ou le mariage de sa fille justifient une consuetudo generalis qui merito est $a b$ omnibus servanda ${ }^{61}$. C'est dans la pratique que les abus sont innombrables, abus qu'entraîne un évident manque de moyens financiers. La pauvreté de la noblesse provençale, inlassablement soulignée, est le leit-motiv de notre juriste. De cette pauvreté, découle l'esprit généralisé d'avarice dans lequel sont traités les tenanciers et les habitants de la plupart des seigneuries ${ }^{62}$.

Une impressionnante série d'exactions et d'injustices fournit autant d'exemples à la démonstration d'Antiboul. Ainsi la faculté d'abandonatio des fonds est iniquement refusée aux homines castrorum, bien qu'elle ne constitute nullement une infraction au ban, ainsi que l'allèguent ceux qu'inspire leur seule cupidité ${ }^{63}$. De même, l'inopia de ces domini les pousse fréquemment à concéder des avantages fiscaux contre quelque travail ou une somme d'argent ${ }^{64}$. Et ces nobiles misérables et intéressés concèdent quotidiennement des assensoymenta, des franchises de tout ordre, mais les monnayent sévèrement auprès des bénéficiaires ${ }^{65}$, ce procédé est particulièrement employé à l'égard des malservi, sur qui reposent les charges les plus lourdes en bien des lieux ${ }^{66}$, et notamment in terra diabolica montanorum ${ }^{67}$. Autre hypothèse courante, le seigneur

60 F.-P. Blanc, L'apparition du droit régalien d'anoblissement en Provence au XIIIe siècle. Provence historique, t. XXIII (1973) (Etudes [...] Edouard Baratier), p. $90-91$.

61 Tract. III, § 94 , fo 28 , c. 1.

62 Tract. IV. 2 , $\S 7$, fo 36 vo, c. 1:,,[...] avaritia itaque dominorum temporalium castrorum cohibetur et reprehenditur [...]".

${ }_{63}$ Tract. IV. 4, $\S 202$, fo 51 , c. 2 : ,[...] ad argumenta dominorum quae non a jure, sed a cupiditatis fonte procedunt, responsio est facilis [...]".

${ }_{64}$ Tract. IV. 4 , $\S 177$, fo $49 \mathrm{vo}$, c. 2 :,,[...] do tibi franchisiam, ut mihi facias quolibet anno tot operas, vel aliquid aliud, vel ut mihi des 25 libras, quod saepe hodie evenit in provincia propter inopiam dominorum castrorum, qui ut pluribus egestate laborant et pecunia egent [...]".

${ }_{65}$ Tract. IV. $4, \S 145, \mathrm{f}^{\circ} 47 \mathrm{v}^{\circ}$, c. 2 :,,[...] raro autem fiunt seu conceduntur hujus modi immunitates hodie a baronibus seu nobilibus liberaliter. Sed semper habent inde pretium seu meritum, et specialiter nobiles acorsati et indigentes: et talia quotidie fiunt in provincia et appellantur vulgariter assensoymenta [...]". De fait, les profits seigneuriaux étaient fréquemment avancés pour justifier les requêtes en affranchissement: cf. R. Aubenas, Le servage à Castellane au XIVe siècle, „Rev. histor. de droit", 4e s., 16e année (1937) p. 88, n. 2.

${ }_{66}$ Sur les obligations des malservi, cf. R. Aubenas, op. cit., p. 83-86; C. Samaran, Note sur la dépendance personnelle en Haute-Provence au XIVe siècle, „Annales du Midi" t. LXIX (1957) p. 229-236; G. Duby, Recherches récentes sur la vie rurale en Provence au XIVe siècle. Provence historique, t. XV (1965) p. 108.

${ }_{67}$ Tract. IV. 4, § 152 , fo 48, c. 1:, ,[...] castrum [...] ubi sunt homines seu rustici valde malservi ac multis gravibus et oneribus onerosis astricti, sicut in provincia in plerisque locis est [...]". En fait, des charges comme les corvées tendaient alors 
tire un injuste profit des amendes dont il élève considérablement le montant 68. Plus féroces encore sont les officiers banniers, envers lesquels Antiboul ne trouve pas de termes assez forts, voire d'injures, pour exprimer son indignation: vils, abjects, infâmes, ribauds, ces agents des „,barons" ne perdent aucune occasion pour pressurer la paysannerie, intéressés qu'ils sont à élever par tous les moyens les produits féodaux ${ }^{69}$.

Non content de fustiger ainsi les tares de la noblesse provençale et de ses serviteurs, l'auteur s'en prend volontiers à cartains praticiens du temps, accusés d'incompétence: témoin ce peritus in jure, id est in brodio, appelé par les rustici du Cannet des Maures pour les conseiller dans un litige qui les opposait à des possesseurs de fonds originaires $\mathrm{du}$ village du Luc: la verve du juriste s'exerce sur ce personnage, qui tamen non credebat fabam esse nigram, mais ignorait la distinction entre impôts réels et personnels ${ }^{70}$. Indépendamment des pauci qui per viam veritatis ambulant, tam bene noverunt viam trahendi aurum et argentum $^{71}$, la critique vise également les miseri notarii qui ne se donnent pas la peine de consulter plus compétents qu'eux et c'est sans aucune sympathie qu'est mentionnée l'exemption fiscale dont prétend bénéficier le notaire des Arcs ${ }^{72}$.

Dans certains cas, l'appel d'Antiboul en faveur d'une justice plus exacte s'élargit aux dimensions de la société du temps. Ainsi le sort du vassal, celui de l'emphytéote et celui du censitaire sont assimilés face aux prétentions du concédant, qui exige fréquemment plus que son dû en excipant seulement du tempus écoulé, et non pas du titulus ${ }^{73}$. Plus remarquable encore est l'opposition entre divites, mediocres et pauperes que le texte établit, en prenant pour exemple la perception d'un fouage

à y disparaître: cf. G. Duby, Note sur les corvées dans les Alpes du Sud en 1338, Etudes [...] Pierre Petot, Paris 1959, p. 141-146.

68 Tract. IV. 4 , § 197 , fo 51 , c. 1 .

69 Tract. IV. 4, § 207 , fo 51 vo, c. 1 : ,[...] cum bannerii hodierni non praefulgeant aliqua dignitate vel honestate, immo viles et abjectae personae ad banneriae officium hodie statuuntur, ut de facto videmus ubique [...]”. Ibid., § 209: „,[..] et ita repellerentur hodie quasi omnes bannerii de provincia, cum vel omnes, vel in majori parte, percipiant partem in bannis, et sunt vilissimi et ribaldi, nullusque bonus homo, vel honestus, illud officium assumit, immo respuit, et recusat velut vilissimum et infame facti infamia: ut notorio de facto ubique videmus maxime in castris et villis baronum". Souvent du reste ces officiers étaient recrutés parmi les notaires: cf. P.-L. Malausséna, La vie en Provence orientale aux XIVe et XVe siecles, Th. Droit, Nice-Paris 1969, p. 39.

${ }_{70}$ Tract. IV. 2 , $\S 35$, fo 34 , c. 2 : ,[...] verumtamen ipse decisionem suam nunquam intellexit, nec scivit quid diceret [...] et cum [...] partes interrogarent ipsum, quae sunt personalia et quae realia, respondit, quod pro uno equo centum librarum hoc non diceret, et forte ideo dicit, quia nesciebat, sed tamen bursam suam injuste ferravit [...]".

it Tract. IV. 4 , § 126 , fo $46 \mathrm{v}^{\mathrm{o}}$, c. 2.

i2 Ibid., § 125 .

73 Tract. IV. 4, § $86-87$, fo 44 vo, c. 2: ,[...] quia vero saepe contingit, quod dominus a vassallo, dominus ab emphyteote, dominus a colono censito pro possessionibus quas tenent sub eo, plus exigit quam sibi debeatur [...]". 
à Trans: s'appuyant sur les statuts de la baylie de Fréjus promulgués en $1235^{74}$, les premiers réclament une imposition fixe par feu, tandis que les autres demandent à contribuere secundum valorem bonorum. Antiboul tranche en faveur de la deuxième interprétation: pro pauperibus teneo ${ }^{75}$.

En conclusion, il nous paraît permis de considérer le traité de muneribus comme l'une des oeuvres les plus originales des juristes méridionaux du XIV ${ }^{\text {e }}$ siècle. Le style n'est certes point exempt de défauts, et notamment de redites. Désordonné presque toujours, parfois verbeux, Antiboul s'emporte avec facilité; au surplus il tire quelque vanité des circonstances où a été louée sa compétence ${ }^{76}$. Sa plume n'en est pas moins d'une rafraîchissante vivacité, et la richesse des exemples qu'elle n'hésite pas à donner l'éloigne de toute monotonie: qu'il s'agisse de modèles empruntés outre-Rhône, comme ces Narbonnais installés à Béziers ${ }^{77}$ ou qui y détiennent des immeubles ${ }^{78}$, ou encore comme la triple titulature du comte de Foix, comte de Pallars et dominus Biarni ${ }^{79}$, ou qu'il s'agisse de circonstances particulières à des localités provençales, comme Tarascon ${ }^{80}$, Grasse ${ }^{81}$, sans oublier Draguignan ${ }^{82}$, Le Cannet ${ }^{83}$ et les villages d'alentour qu'il connaît si bien ${ }^{84}$, les pages du Tractatus abondent en notations concrètes qui permettent la confrontation permanente de la doctrine et de la pratique.

L'homme était surtout sensible à la misère rurale et aux injustices sociales du temps: la langue forte et savoureuse, que tamise à peine un latin imparfait, laisse percevoir une hostilité marquée à l'encontre d'un système féodal détourné de ses origines, hostilité dont la violence croissante donne à la fin de l'ouvrage le ton d'un pamphlet. Que l'auteur

74 Ed. F. Benoit, Recueil des actes des Comtes de Provence appartenant à la maison de Barcelone, Alphonse II et Raimond Bérenger V (1196-1245), t. II, Monaco-Paris 1925, p. 323-337.

75 Tract. IV. $2, \S 48$, fo 35 , c. 2.

76 Tract. IV. $2, \S 65$, fo 36 , c. $1:,[\ldots]$ de ista quaestione plene tunc allegavi, quae allegationes fuerunt visae Avenion. per magnos advocatos et laudatae, et introductae in consilio regio, ubi fuerant multi doctores et jurisprudentes".

77 Tract. IV. I, $\S 16$, fo 20 , c. 1.

78 Tract. II, $\S 21$, fo $21 \mathrm{vo}$, c. 1.

79 Tract. III, $\S 82$, fo 27 , c. 1 . - Sur les origines de cette titulature, cf. P. Timbal, La tutelle dans la famille des comtes de Foix au XIVe siècle, „Recueil [...] de droit écrit", fasc. I (1948) p. 69-76.

80 Tract. I, $\S 35$, fo $20 \mathrm{v}^{\mathrm{o}}$, c. 1 .

81 A propos de l'imposition sur les langues de boeuf: Tract. IV. 2, § 11, fo 33 vo, c. 1 .

82 Albergue de la Saint-Michel: ibid., § 16. - Composition entre le comte et les habitants: ibid., $\S 49$, fo 35 , c. 2 . etc.

83 Traitant des fontaines publiques converties à un usage privé, il cite la fontaine dite Frufranca, apud Torriolam (auj. Taurelles, lieu-dit. comm. Le Cannet des Maures, en direction du Luc): Tract. IV. 4, § 107, fo 45 vo, c. 2.

84 Carcès, Cotignac (Tract. IV. 2, § 35, fo 34, c. 2), Le Luc (ibid., § 36 ); il a plaidé pour les communautés, ou conseillé les habitants de Lorgues, du Muy, de Bargemon (Tract. IV. 4, §53, fo 42 vo, c. 2), de Flayosc (ibid., $\S 71$, fo 44 , c. 1) etc. 
ait été profondément marqué par l'enseignement de Brémond de Montferrier ne fait aucun doute: trop de ses critiques forment contrepoint aux plaintes exprimées par son condisciple Pierre Jame ${ }^{85}$. Mais, pur provençal ${ }^{86}$, Antiboul sait, peut-être mieux que ne le fait, dans un style plus contenu et plus technique, l'auteur de l'Aurea practica, transmettre sa flamme et, à l'envers de tant de juristes méridionaux plus occupès à faire carrière ou à arrondir leur patrimoine qu'à rappeler les principes d'une saine justice, lancer un cri dont il mesure lucidement la portée ${ }^{87}$ et auquel les modernes, à travers les travaux qui ont montré pour l'époque la fragilité d'une économie de pauvre rendement ${ }^{88}$, ne peuvent rester insensibles.

${ }_{85}$ Ainsi lorsque ce dernier, décrivant le caractère patrimonial de la justice féodale, conclut: ,,[...] et sic utilitas pecuniaria magis in jurisdictione spectatur et inspicitur quam virtus justitiae". (éd. cit., fo 87 vo).

${ }_{86} \mathrm{Ce}$ trait explique sans doute que, traitant de la maxime rex est imperator in regno suo, Antiboul écrive de jure tamen subest imperio (Tract. IV. 4 , § 98 , fo 45 , c. 2), tandis que Jame, après un long examen de la question, déclare non subest imperatori romano nec de jure nec de facto (ed cit., fo $48 \mathrm{v}^{\mathrm{o}}$ ).

${ }_{87}$ Tract. IV. 4 , $\S 202$, fo 51 , c. 2 : , ,[...] ista solutio et opinio mea non bene cantat pro dominis castrorum, nec placeret eis, si eam audirent. Sed ego non curo cum talis sit veritas juris".

${ }_{88}$ Selon l'expression de J. Glénisson, L'enquête pontificale de 1373 sur les possessions des hospitaliers de St. Jean de Jérusalem, „Bibl. Ecole des Chartes”, t. CXXIX (1971) p. 95. 